\title{
INVESTIGATION OF THE METHOD OF IMPROVING THE INTERFERENCE PROTECTION OF AXISYMMETRIC ANTENNAS IN THE ANTERIOR SEMISPACE
}

\author{
V.I. Magro*, V.M. Morozov \\ ${ }^{1}$ Oles Honchar Dnipro National University, Dnipro, Ukraine \\ e-mail:magrov@i.ua
}

The method of improving the interference protection of axisymmetric antennas is considered. An experimental study of the focusing properties of a zonal antenna is carried out in this work. Such a zonal antenna acts as a shading screen and is located in the aperture of an axisymmetric antenna. The combination of a shading screen with an axisymmetric antenna can significantly reduce the level of side lobes in the anterior semi-space, and as a result, significantly improve the immunity of the antenna system. A zonal antenna for $40 \mathrm{GHz}$ frequency is manufactured. The field distribution along the axis of symmetry for different numbers of rings in the antenna is experimentally investigated. The number of rings varied from 1 to 5 . The focusing properties of the zonal antenna are studied at the change of operating frequency. The position of the focus is investigated when the working frequency changes in relation to the calculated frequency. It is established that the experimentally found focal point does not coincide with the focal point found by geometric optics. For a zonal antenna with three rings, the difference between the experimentally found focus and the focus found by the law of geometric optics is $0.1 \%$. The distribution of the field in planes perpendicular to the focal line is investigated. It is established that an increase in the number of rings in a zonal antenna to more than five leads to diffraction effects which impair the focusing properties of the zonal antenna. At the same time there is a "deliquescence" of the wave beam in the focus. These studies allow us to determine the boundaries of the working frequency of a complex antenna system.

Keywords: axisymmetric antenna, interference protection, zonal antenna, focus, field distribution.

Received 10.09.2019; Received in revised form 13.10.2019; Accepted 15.10.2019

\section{Introduction}

Although the principles of building a zonal antenna are known for a long time, however, the peculiarities of this design work is still not sufficiently investigated [1-4]. This is due to the fact that the principle of antenna construction is based on the laws of geometric optics. This antenna can be used as an independent design, but may be a part of more complex antenna designs [5]. In the latter case, the diffraction antenna serves as the limiter of the lateral radiation of an axisymmetric antenna.

There are many reasons that cause the side radiation of the antenna and thus worsen the interference protection of the antenna. Therefore, ways to combat this negative phenomenon are quite diverse. There are ways to reduce the antenna's side radiation in some corner area, regardless of the causes of this radiation. Let us consider the classification of negative side radiation and the reasons that give rise to them.

1. Aperture diffraction beams. They are generated by currents on the antenna's working surfaces, which are determined by the distribution of the amplitudes and phases of these currents, as well as their polarization structure. It is possible to reduce the indirect radiation of this type dramatically by choosing the necessary distribution of currents.

2. Dispersing energy on the irradiator and elements of its attachment. The method of combating this lateral radiation is the transition to a non-symmetric antenna.

3. Side beams of irradiator of an axisymmetric antenna. The methods of struggle are optimization of the radiation pattern of the irradiator or the installation of additional structures on an axisymmetric antenna.

One of the versions of additional constructions that can be installed on an axisymmetric antenna is the annular shading screen. They allow us to reduce lateral radiation significantly. However, in the literature there is no study of the frequency characteristics of a zonal antenna. It is known that an axisymmetric antenna belongs to a class of frequencyindependent antennas, while it is very important to know the limits of the working range of 
the entire antenna construction with taking into account the characteristics of the zonal antenna. Therefore, the purpose of this paper is to study the frequency characteristics of the zonal antenna depending on the number of elements in the antenna.

\section{Experimental procedure}

When calculating the size of the zonal antenna, the laws of geometric optics are used. As a focus, a point is found that is at a distance $f$ from the plane of the antenna. The signals emitted by the mid-circles of the zone antenna will coincide in phase with the focus at the following values of distances between the edges of the circles and the focus. In the general case:

$$
l_{m}=f+\frac{(2 n-1) \lambda^{2}}{4}, \mathrm{n}=1,2,3 \ldots
$$

Thus, the signal emitted by this circle at the focus will be in phase with a signal emitted by the disk center. On the surface of the second circle will be a circle, the distance from which to the focus is equal to $f+2 \lambda$, on the surface of the third circle $f+3 \lambda$, etc. All these signals will be in-phase in focus.

To study the frequency properties of the zonal antenna, an antenna designed to operate at a frequency of $40 \mathrm{GHz}$ is installed. The quantity of elements in antennas $\mathrm{N}$ was compiled from one to five. The estimated focal length of the antenna $f$ is $100 \mathrm{~cm}$. The zone antenna is irradiated with a plane electromagnetic wave. The incident electromagnetic wave is linearly polarized in the vertical plane.

\section{Results and discussion}

A study of the focusing properties of a zonal antenna for a different number of elements in the antenna is conducted.

Fig. 1 shows that the difference between the calculated and actual focus for an antenna with one ring is $15 \mathrm{~cm}$, this error is $10 \%$. For an antenna with two and three rings, the difference is $1 \mathrm{~cm}$ that is $0.1 \%$. For four rings, this error is $2 \mathrm{~cm}$ or $0.2 \%$. For an antenna with five rings, the calculated focus and the real one match. A further increase in the number of rings in the antenna leads to various diffraction effects, which reduce the focal capacity of the zonal antenna.

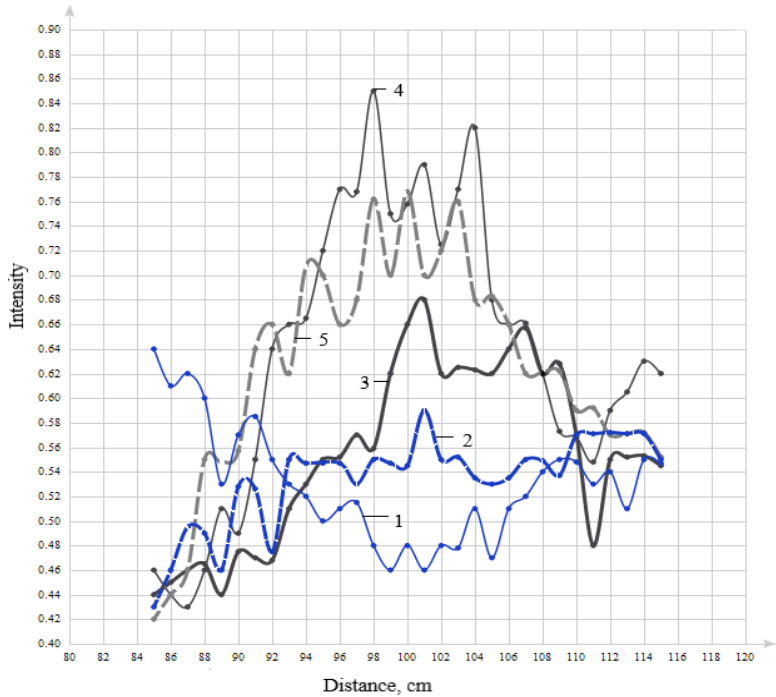

Fig.1. Investigation of the field intensity along the antenna axis $(\mathrm{N}=1,2,3,4,5)$ at $f=40 \mathrm{GHz}$. 
A study of the focusing properties of the zonal antenna at the change of operating frequency is performed. The operating frequency varied from $39 \mathrm{GHz}$ to $41 \mathrm{GHz}$. The results of the study of focusing properties at a frequency of $39.5 \mathrm{GHz}$ are shown in Fig. 2 and Fig. 3. It is seen that the longitudinal distribution of the intensity of the field has a clearly expressed maximum for 3 rings, but this maximum intensity is shifted relative to the actual focus by $10 \mathrm{~cm}$. For an antenna consisting of one ring, it can be seen that the maximum intensity is observed at a distance of $85 \mathrm{~cm}$ and the difference between the calculated focus and the actual one is $15 \%$. For an antenna consisting of two rings the real and estimated focuses are the same.

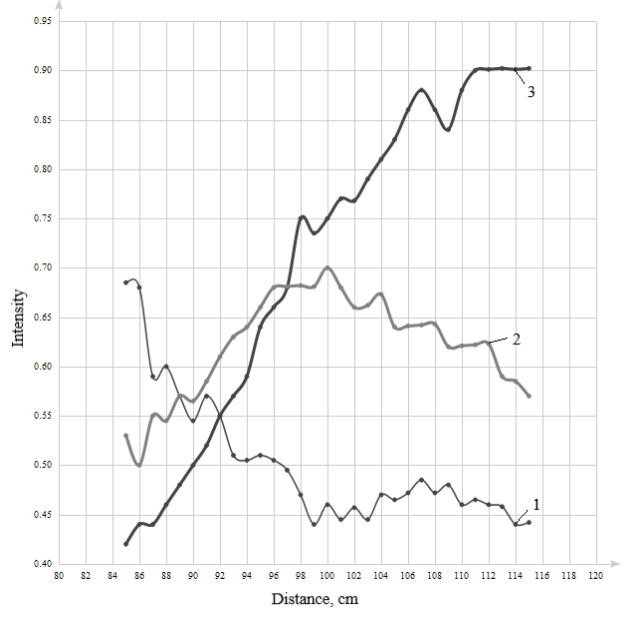

Fig. 2. Investigation of the field intensity along the antenna axis $(\mathrm{N}=1,2,3)$ at $f=40 \mathrm{GHz}$.

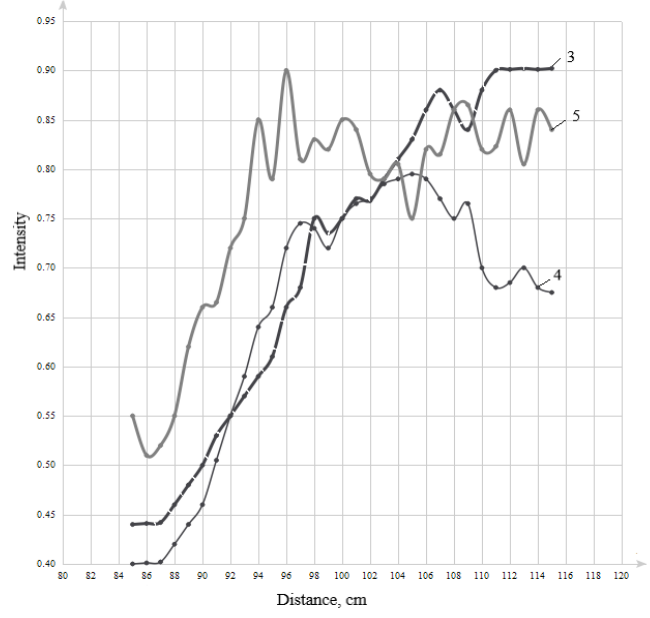

Fig. 3. Investigation of the field intensity along the antenna axis $(\mathrm{N}=3,4,5)$ at $f=40 \mathrm{GHz}$.

For an antenna of four rings it can be seen that the difference between the actual focus and the calculated focus is $5 \mathrm{~cm}$ or $0.5 \%$. So, we can draw the following conclusion about the main tendencies that occur when the operating frequency is less than the calculated frequency: the actual focus at a reduced operating frequency and with an increase in the number of rings is shifting from the plane of the antenna. However, for two rings, the actual focus is the same as the calculated one, which is calculated for a frequency of $40 \mathrm{GHz}$. An increase in the number of rings in the antenna leads to an offset of the focus from the plane of the antenna by about $0.5 \%$. An additional increase in the number of elements in the antenna can lead to resonant effects that impair the focusing properties of the zonal antenna.

From the graph for one ring in Fig. 4 it is seen that the maximum intensity is at a distance of $86 \mathrm{~cm}$, which is a difference of $14 \%$ relative the calculated focus for a frequency of $40 \mathrm{GHz}$. For two rings, the maximum intensity is at a distance of $102 \mathrm{~cm}$, the difference is $0.2 \%$. For three rings it is seen that the focus is at a distance of $102 \mathrm{~cm}$, it is shifted by $0.2 \%$. For the four and five rings this difference is already $0.1 \%$. Thus, the main trends observed with an increase in operating frequency up to $40.5 \mathrm{GHz}$ are: for the number of rings from 2 to 5 , the actual focus is at a range of $0.1-0.2 \%$ of the focal point, which is designed for a frequency of $40 \mathrm{GHz}$. Increasing the number of rings leads to the maximum approximation of the real focus to the calculated one.

The results of studying the focusing properties of the antenna at $40.5 \mathrm{GHz}$ are shown in Fig. 4 and Fig. 5.

If the operating frequency is $41 \mathrm{GHz}$, then the best focusing properties of the zonal antenna are observed for the number of rings four and five. At the same time for the four 
rings the difference between the tricks is $0.3 \%$, for the five rings $-0.5 \%$. As in the previous case, an increase in the number of rings leads to the maximum approximation of the real focus to the calculated one.

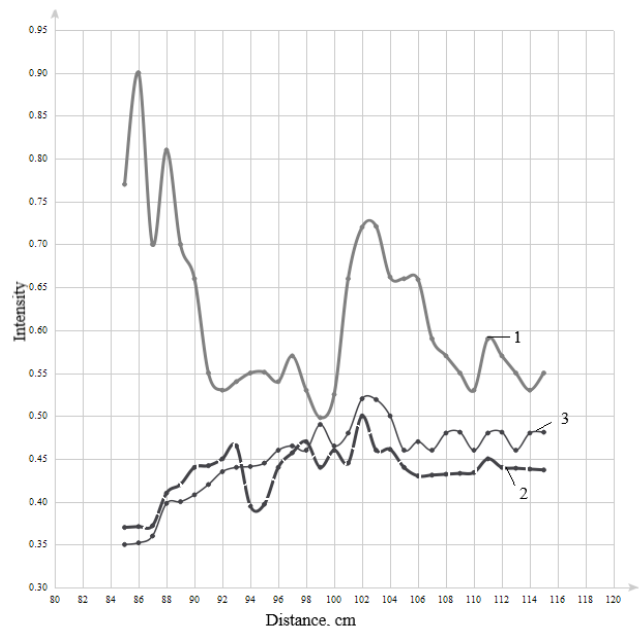

Fig. 4. Investigation of the field intensity along the antenna axis $(\mathrm{N}=1,2,3)$ at $f=40.5$

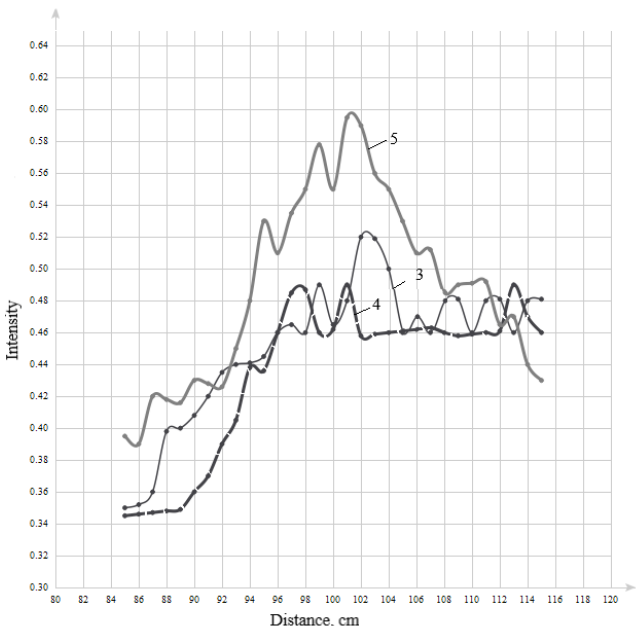

Fig. 5. Investigation of the field intensity

For an antenna of four rings at a working frequency of $39 \mathrm{GHz}$, the difference between the focus is $0.1 \%$, for five rings $-0.5 \%$. In addition, the actual focus is closer to the plane of the antenna than the calculated focus (Fig. 6).

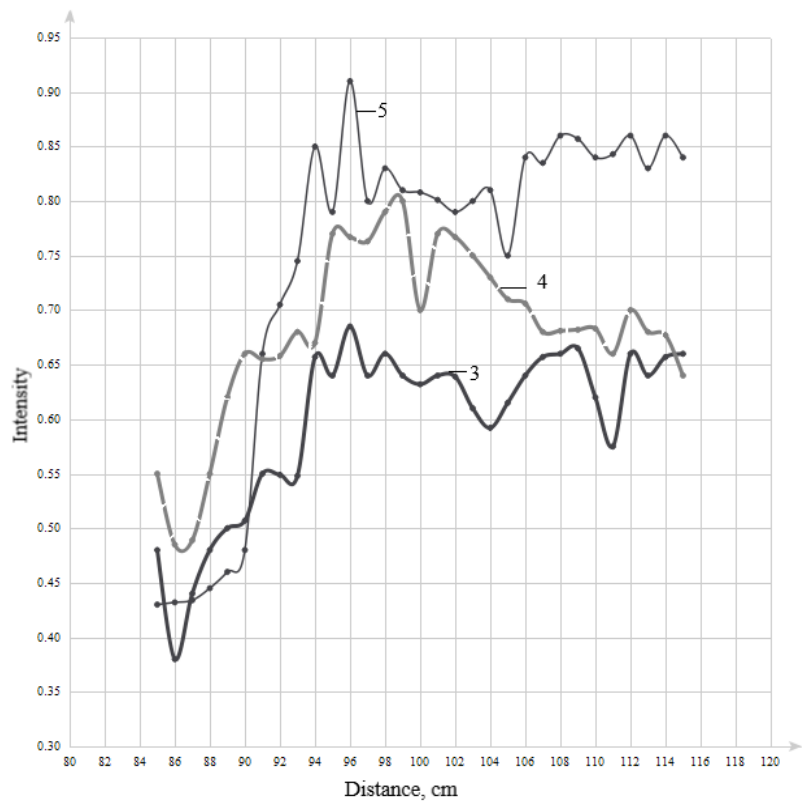

Fig. 6. Investigation of the field intensity along the antenna axis $(\mathrm{N}=3,4,5)$ at $f=39 \mathrm{GHz}$ 
The study of field distribution in the section located at the point of the calculated focus at a frequency of $40 \mathrm{GHz}$ is conducted. The transverse shift relative to the axis of symmetry of the antenna is denoted as L.

The results of this study are shown in Fig. 7 and Fig. 8.

For five rings, the highest signal level and the best focusing of the wave beam are observed. A similar study is conducted in sections, which are located at distances of $2 \mathrm{~cm}$ on both sides of the calculated antenna focus. For these cross-sections, it is found that the maximum signal is observed for four rings.

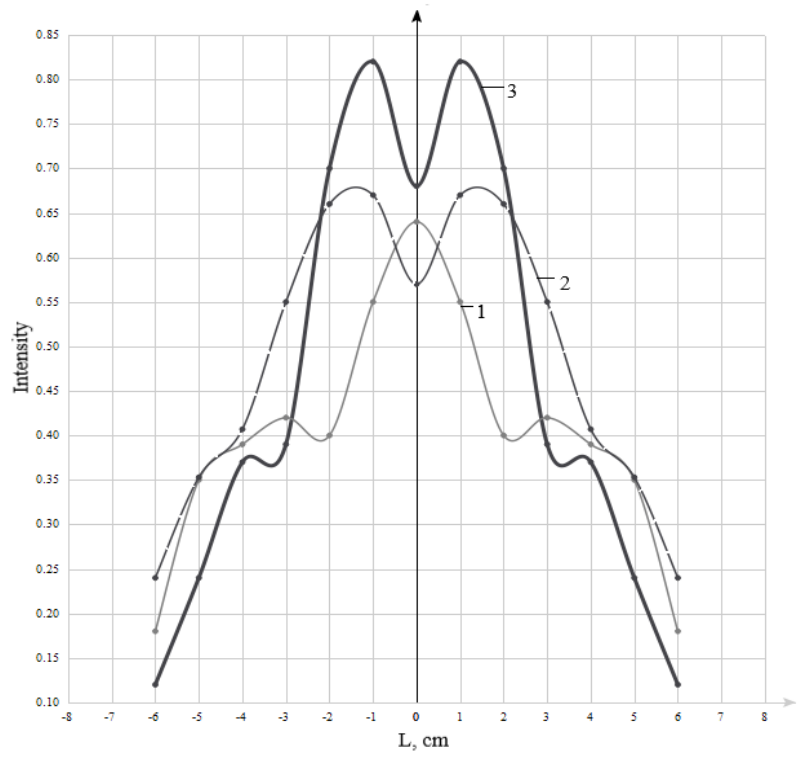

Fig. 7. Distribution of signal intensity in the section of the calculated focus at $f=40 \mathrm{GHz}(\mathrm{N}=1,2,3)$.

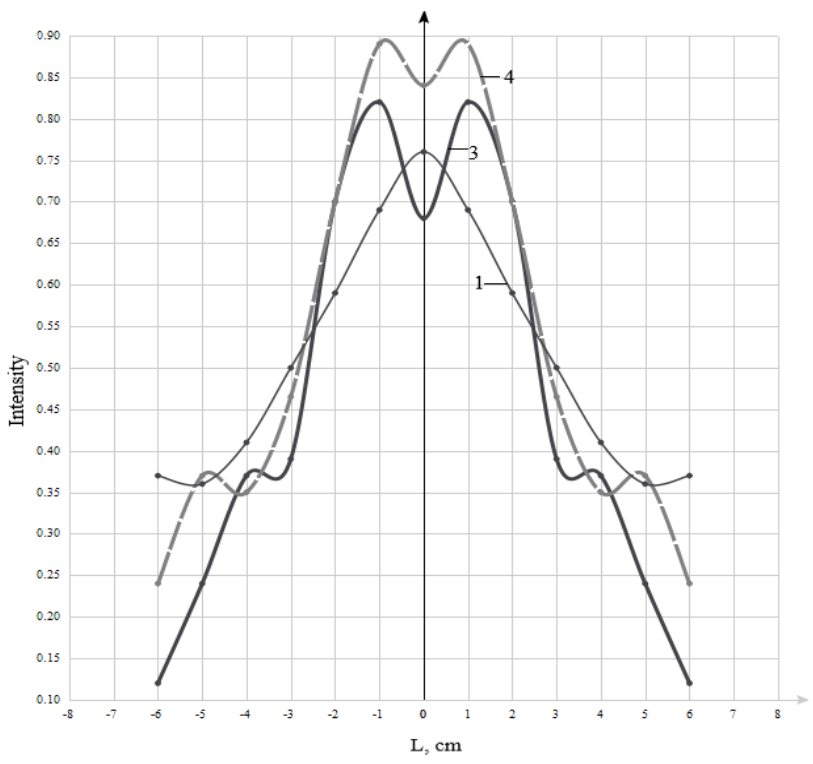

Fig. 8. Distribution of signal intensity in the section of the calculated focus at $f=40 \mathrm{GHz}(\mathrm{N}=3,4,5)$. 


\section{Conclusions}

Thus, we can draw the following conclusions:

1. The use of diffraction screens together with axisymmetric antennas can significantly reduce the level of side lobes of the axisymmetric antenna, that is, significantly increase its noise immunity.

2. The number of rings in the diffraction screen depends on the width of the working frequency band of the antenna system. If the width of the working frequency band is very small, then it is advisable to use screens with five or more rings. When expanding the working frequency band of the antenna system, it is necessary to reduce the number of rings in the diffraction screen. But reducing the number of rings reduces the noise immunity of the antenna system.

\section{References}

1. Minin, I.V. Basic principles of Fresnel antenna array. Lecture notes electrical engineering / I.V. Minin, O.V. Minin. - Springer, Berlin, 2008. - Mode access: https://www.springer.com/gp/book/9783540795582

2. Ojeda-Castanada, J. Selected papers on zone plates / J. Ojeda-Castanada, C. Gomez-Reino. - SPIE Press. - 1996. - 486 p.

3. Magro, V.I. Modification of radiation characteristics in Fresnel antenna / V.I. Magro, V.M. Morozov // Conference Proceedings of Second International Workshop "Ultrawideband and Ultrashort Impulse Signal" (UWBUSIS'2004). - Sevastopol, Ukraine, 2004. - P. 273 - 275.

4. Magro, V.I. Investigation of radiation characteristics of Fresnel antenna / V.I. Magro, V.M. Morozov // Conference Proceedings of 9th International Kharkiv Symposium on physics and engineering of microwave, millimeter and submillimeter waves (MSMW'2016). - Kharkiv, Ukraine. - 2016.

5. Yampol'skiy, V.G. Antenny i EMS / V.G. Yampol'skiy, O.P. Frolov. - Moskva: Radio i svyaz', 1983. - 272 s. 